\title{
The potential impact of dietary supplement adulteration on patient assessment and treatment from a healthcare provider's perspective
}

\author{
Gary Gabriels* and Mohamed Irhuma \\ Department of Pharmacy and Pharmacology, Faculty of Health Sciences, University of the Witwatersrand, \\ Johannesburg, South Africa \\ *Corresponding author, email: gary.gabriels@gmail.com
}

Dietary supplements have been manufactured and supplied to the market with the objective of enhancing the overall health of the general population and optimising the performance of athletes. The perceived intention of dietary supplementation is to increase the nutritional content of a normal diet, and to fill a dietary need and/or presumed deficiency. The usage and popularity of dietary supplements, however, raises concerns from a health benefit and risk perspective. Moreover, safety and efficacy of these supplements have generally not been established by the Medicines Regulatory Authorities, both nationally and internationally. The exponential increase in supplement sales can, however, be attributed to aggressive marketing by manufacturers, rather than the development of more effective nutritional supplements. These supplements may contain adulterated substances that may potentially have harmful short- and long-term health consequences for the patient. In addition, a large spectrum of drug interactions may render the use of such supplements risky, without proper medical and scientific assessment.

Keywords communication, curriculum, life-style, physician-patient relationship

\begin{abstract}
Introduction
A healthcare practitioner is a professional who practises medicine, restores health or maintains good wellness for the patient. In recent times these elements have been applicable to the self-preservation also of the practitioner's health and wellness. The practitioner is concerned with promoting, maintaining or restoring better health condition through the study, diagnosis, prevention and treatment of disease, injury, and other physical and mental impairments. The important consideration in this paper is the obligation of the healthcare provider to conscientiously take into consideration, through appropriate and rigorous assessment, whether the patient has or consumes dietary supplements when introducing dietary-together with lifestyle -intervention, and where in particular medicine will be prescribed for treatment but not exclusively so.
\end{abstract}

\section{Patient autonomy, practitioner's 'pledge' and professional obligation}

The current climate of medicines in health and wellness provision has been the conversion of the 2500 -year-old Hippocratic Oath to a pledge that is becoming its contemporary successor. ${ }^{1,2}$ The most pronounced feature in the pledge is patient autonomy $^{1}$, and incorporates the humanity of physicians, which requires awareness by the healthcare provider to maintain his/ her own health ${ }^{3}$, well-being and abilities in order to provide care of the highest standard. ${ }^{1,3}$ The 'Physician's Pledge' thus more accurately reflects the challenges and needs of the modern medical profession and potential practice complexity. ${ }^{3}$

The new obligation for physicians through the pledge is to share knowledge for the benefit of their patients, and for the advancement of health care. ${ }^{1}$ The previous oath formed part of the medical professional code and was legally binding. The 'pledge', however, is intended to be an ethical code for all physicians, and by extension for the broader healthcare profession. ${ }^{1}$
The purpose via its implementation is to strengthen the respective healthcare professions, with determination to maintain the highest standard of health care for patients. ${ }^{1}$ The pledge makes it a mandatory responsibility to teach and provide knowledge to the next generation of physicians. ${ }^{3}$ Its importance lies in sharing ${ }^{1}$ and in continued teaching and knowledge ${ }^{3}$ acquisition, the more so with specific reference to keeping abreast of the prevailing landscape of dietary supplement consumption, and the impact this could have on the health/well-being of the physician and patient.

This responsibility may not be apparent but in the absence of a more discerning regulatory environment worldwide with regard to dietary supplements and adulteration/contaminants in such products, the practitioner would be required to be more vigilant. The further burden of vigilance is the reality and probability of ongoing changes in dietary supplement products due to aggressive marketing ${ }^{4}$, and thus the possibility of these products' composition frequently changing. Specific awareness should be placed on the potential adverse impact this may have on patients' well-being via embedded contaminants/adulterants that may be in these products but undeclared on the label. ${ }^{5}$

Perceived patient autonomy and possible non-disclosure of dietary supplement consumption becomes an important part of the assessment and treatment plan, and could become a new challenge in respect of the physician-patient relationship. For the practitioner, it is critical that questions of consumption of such products not be omitted, regarding what may be/is allowed to be prescribed. Specifically this relates to the probability of adulteration/contamination of these products, possible interactions with current chronic therapy, and lack of rational indications, all of which could contribute to adverse effects and a negative healthcare impact. Further, added awareness on the part of the practitioner becomes important if the practitioner consumes supplements, as this may contribute to 
distorted practitioner physiology, potentially clouding judgement and decision-making.

\section{Health and wellness providers: communication at the centre}

Communication, in the form of patient education, remains at the core of this new paradigm and/or complexity for practitionerpatient relations, ${ }^{6}$ in particular where anger, anxiety or depression is evident. ${ }^{7}$ For healing to occur in a dignified manner, an environment that is stable and trusting with good interpersonal relations is required. ${ }^{6}$ This would enable the goal of practising medicine or allied disciplines as a profession to succeed $^{6}$ in a modern environment. The environment would thus facilitate free exchange of information encompassing both verbal and non-verbal interactions, ${ }^{7}$ and should include the patient in decision-making towards 'healing'. ${ }^{6}$

The practitioner's relationship goals can best be served through various communication approaches: (i) the shared model of communication, ${ }^{6}$ which is and would be the best fit for communication in the twenty-first century between physician and patient, (ii) the paternalistic historical model, which involves physician-patient relations that encompass dependency on physician authority, and, (iii) the informed model, which involves a partnership between physician and patient that is based on a division of labour. ${ }^{6}$

The evolution of the shared model that will best serve the nexus of engagement and contribute to the knowledge-cycle in a patient-centred strategy strives to understand the patient's illness within a combined biological, psychological, clinical and social perspective. ${ }^{6}$ The model takes into account the patient's individual experience, personal meaning of illness ${ }^{6}$ and joint agreement in decision-making. This may inevitably be stressful and challenging for both parties ${ }^{7}$, and more so in the context where identified dietary supplements form part of the diet and consumption consequences ${ }^{6}$ may arise. This model will help further in the absence of a rigorous regulatory framework and enforcement capability, pertaining to the dietary sector broadly at the consumer level, and can assist in the patient consumption experience.

Communication thus contributes to a core clinical assessment in terms of the emotional response, well-being, practice satisfaction, source of motivation, reassurance and support for the respective healthcare professions and the respective patient. ${ }^{6,7}$ Further, physicians should be aware that their own sense of what constitutes a good encounter may differ from that of many patients, especially due to cultural differences. ${ }^{7}$ Communication diagnostics and scrutiny therefore need to embrace more than before: the perceived requirement by the prevalence of consumption, and the demand for dietary supplements by consumers and patients. What may not be initially apparent is the contribution of adverse effects profile, which may be related to consumption and possible drug-supplement interaction, and which should be considered in terms of contamination and physician prescriptions for treatment. Therefore, practitioners need to consider the possibility of unrecognised substance abuse or a personality disorder. ${ }^{7}$ The unrecognisable substance abuse may very well be based on the patient's lifestyle consumption of dietary supplements that have embedded substances or prohibited substances that are not declared by the manufacturer ${ }^{5}$ on the product label. These lifestyle products may be the contributing factor to the anger, anxiety and depression, ${ }^{8}$ which may occur during the physician-patient engagement. In order to solve the patient's problem or ailment, it may be necessary to incorporate the withdrawal of the consumption of the dietary supplements, be this gradual or immediate, to ensure improved physician-patient communication, or to solve the problem.

\section{Prior knowledge of the patient, and physician decision-making}

The work of Hjortdahl (1992), ${ }^{9}$ in a Norwegian study, indicates that prior knowledge about a patient by the healthcare practitioner was clinically useful to make a viable diagnosis. This is particularly the case where there are psychosocial problems. ${ }^{9}$ In the absence of this history, when the patient has embarked on a new lifestyle modification, such as the consumption of dietary supplements, special diagnostics knowledge is important, when patients present with new, unspecific problems, such as fatigue, fever and generalised pain. ${ }^{9}$

Further, where psychiatric disorders are highly prevalent in the community, many patients with a psychiatric morbidity remain unidentified as such in primary care. ${ }^{10}$ Earlier research ${ }^{10}$ also shows that female patients and patients well known to their general practitioners (GPs) have a better chance of being recognised as suffering from a mental illness. ${ }^{10}$ In general, patients' psychological problems are also not inclined to be put forward as a demand for help to their GP, because they might expect treatment with medication rather than counselling or another form of therapy. ${ }^{10}$ The healthcare provider should thus take this into consideration as part of the assessment. It should not be ruled out that the new ailment (e.g. psychiatric disorder) has manifested as a result of the lifestyle modification.

The lifestyle could be the contributory element in DionysianApollonian Duality, the connection being that it is well known that prohibited substances, narcotics and stimulants have the ability to alter a 'state of mind'. ${ }^{11}$ Psychoactive drugs-an example being the opioids, which have sleep-inducing properties, and psychotropic drugs, which alter chemical levels in the brain-have the ability to impact mood and behaviour. ${ }^{11}$ Where these drugs are embedded and often undeclared in the consumed dietary supplement product, they could be linked to the 'darker' elements of the patient personality, which causes the 'shifting states-of-mind', due to distortion from normal physiological and psychological effects. ${ }^{11}$

\section{Lifestyle, consumption and 'pattern' changes}

Undifferentiated and unorganised problems are a common challenge for practitioners; ${ }^{12}$ this may not have extended to dietary supplement consumption as an additional consideration. As an illustration: the upsurge in the use of natural products, in general, includes: (i) the cost effectiveness and availability, (ii) the perceived safety and lack of side effects, (iii) a weakened belief in the efficacy and safety of orthodox drugs, and (iv) the public tendency towards self-medication. ${ }^{13}$ Concern associated with herbal supplement use includes the potential exposure to toxic substances, whether as an expected component of the formulation or as an unintended contaminant. ${ }^{13}$ There is no reason to suspect an offending herbal supplement to be at risk of overexposure to heavy metals, until the consumer shows signs of toxicity. ${ }^{13}$ Thus, it is important for proper clinical evaluation of the patient to determine the use of herbal supplements as part of diagnosis, as the patient may not necessarily declare such use to the physician provider. Evidence from the work of Gabriels et al. ${ }^{14,15}$ on contamination/adulterants in dietary supplements is that of fluoxetine ${ }^{14}$ and melamine, ${ }^{15}$ which may 
potentially have harmful short- and long-term health consequences for the patient. ${ }^{15}$

Fluoxetine, a selective serotonin reuptake inhibitor (SSRI), is being used for the management of major depressive disorders and mood changes. ${ }^{14}$ The presence of fluoxetine as a mood enhancer in dietary supplements becomes a consideration when deciding on treatment with antidepressants and knowledge of an embedded antidepressant in nutritional/dietary supplement product is important. ${ }^{14}$ Specifically for fluoxetine, prescriber caution for treatment in the elderly in particular includes adverse effects, particular neurological effects such as suicidal ideation, agitation, anxiety, insomnia, seizures and insomnia, or sexual dysfunction effects, such as anorgasmia and decreased libido. ${ }^{14}$ The 'early warning signal' by the physician can assist with improved insight into the possible embedded SSRIs when prescribing treatment. ${ }^{14}$

'Scrap melamine' is another example of an adulterant contaminant ${ }^{15}$ that has been shown to be present in dietary supplement products and has been used as a source of non-protein nitrogen, which falsely increases the claimed protein content. ${ }^{15} \mathrm{~A}$ variety of serious adverse effects have been mentioned or associated with melamine intake, such as renal failure and urinary bladder neoplasm. ${ }^{15}$

Thus, for the general practitioner, dealing with the probability of consumption of dietary supplements becomes an essential skill, in knowing or deriving what the patient consumes, and the intended treatment provision, ${ }^{3,} 6$ matched with evidencebased medicine. ${ }^{3}$

\section{Exacerbation of disease biomarkers caused by dietary/nutritional supplements}

Currently there is no treatment to cure neuro-degenerative disorders like Alzheimer's disease (AD) and Parkinson's. For Alzheimer's disease $(A D)$, evidence from a multinational survey ${ }^{17}$ shows that whilst most patients present first to a physician, the eventual diagnosis is more likely to be made by a specialist. $^{17}$ The progressive nature of Alzheimer's disease leads to the destruction of memory and other important mental functions, but this may be caused or exacerbated by physiological imbalance in the patient ${ }^{14}$ as a result of lifestyle or medical requirements $^{14}$ through the consumption of dietary supplements. The aim of the current available pharmacotherapy for these disorders is to prevent the progression of such degenerative illness and to restore some functions in order to promote better quality of life. However, the inner workings of undeclared embedded contaminants/adulterants may cause harm to the patient and also potentially exacerbate the disease state, and thus work contrary to the pharmacotherapy intention.

The cause and/or exacerbation of the disease may be as a result of constituents, often undeclared, that may not be apparent to the physician or others. Early detection and diagnosis, rather than eventual specialist diagnosis, ${ }^{17}$ may well assist the retardation of $A D$ when lifestyle modification is instituted early. This specifically is where suspicion or clarification is focused with respect to dietary supplement consumption. Moreover, a study conducted by Block et al. ${ }^{18}$ found that users who consumed a wide selection of nutritional supplements on a daily basis were more prone to have optimal concentrations of chronic disease-related biomarkers, and be less prone to suboptimal nutrient concentrations, high blood pressure and diabetes when compared with non-users of nutritional supplement. ${ }^{18}$

\section{Common medication, exercise and training, and dietary consumption}

Common over-the-counter and prescription medication may cause side effects if an individual exercises. ${ }^{19}$ The potential side effects would be dependent on the type of medication consumed..$^{19}$ People may generally be aware of caffeine-containing beverages that could elevate the heart rate. ${ }^{19}$ The realisation and suspicion that cold medications, diet pills, allergy remedies and herbal teas ${ }^{19}$ could do likewise may not necessarily be immediately evident, with examples such as bronchodilator, nicotine and thyroid replacement medication use in the context of the exercise. ${ }^{19}$ Further, the same is applicable to a dietary supplements consumption lifestyle, as these products may contain compounds ${ }^{5}$ that could contribute to side effects under normal use conditions, but these effects may be elevated when doing exercise. ${ }^{5}$ The consumption of combinations of dietary supplements, consecutively and/or collectively, could cause a profound increase in heart rate, blood pressure and a decrease and/or varied effects on exercise capacity, including in persons with co-morbidities, especially those suffering frequent anxiety attacks, and in smokers and the elderly. ${ }^{19}$

Healthcare professionals and exercise specialists should therefore be aware of the patient's exercise programme, lifestyle and the effects that medications and potentially tainted products, such as dietary supplements, could have on his/her habitual adjustments. ${ }^{19}$ The healthcare provider should therefore adhere to a holistic, individualistic approach when consulting with a wide range of patients, ${ }^{19}$ whilst utilising medication and exercise guidelines as a tool.

\section{New understanding of 'patterns', and the curriculum}

Research has shown that the dietary supplement industry is not adequately and appropriately regulated, resulting in claims made for the products that may not always be accurate. ${ }^{20}$ The usage and popularity of dietary supplements, however, raises concerns from a health benefit and risk perspective. ${ }^{21}$ Instead, these products' containers could manifest innovative ideas for the business-minded entrepreneur, ${ }^{20}$ in terms of both the content and the information, and the information that is omitted. Presently in sport, health and fitness professionals are faced with the dilemma of their athletes doping and experiencing supplement 'abuse' through dietary supplement consumption. One could argue that it is the responsibility of the team doctor, trainer or conditioning specialist to guide athletes on whether certain products are safe to use or not. ${ }^{20}$ In addition, drafting dietary supplement policies and amended legislation or newly formulated legislation would protect patients, and sportspersons, and also safeguard the manufacturer, distributor and retailer. ${ }^{20}$ Ethical physicians could thus play an important role in providing advice to the patient that would minimise the risk for 'testing positive' as a result of a doping violation. However, work by Starzak et al. ${ }^{22}$ shows a lack of doping awareness by medical professionals, despite the global prevalence of doping in sport. Despite this knowledge, ${ }^{22}$ and the need for doping prevention strategies, information about doping awareness among medical professionals remains limited. ${ }^{22}$ Today, more than ever, elite athletes are motivated to utilise prohibited substances in order to enhance their performance and body image, and by implication their social and psychological standing. ${ }^{22}$

Notwithstanding the continuing rise in the abuse of prohibited substances amongst sports participants, the findings in the study of Starzak et al. ${ }^{22}$ indicate that both doping-related knowledge 
and doping-specific training amongst South African pharmacists, and to a larger extent GPs, is considerably deficient. ${ }^{22}$ In that study, pharmacists and general practitioners in South Africa admitted poor knowledge of doping-related matters, and lacked doping-specific training. ${ }^{22}$ Overall the study found that there was a consensus amongst medical practitioners that they have a role to play in doping prevention. ${ }^{22}$ This highlights the need to review medical professionals' education, curriculum and ongoing training and development in relation to doping in sport. ${ }^{22}$

Whilst it is time for improved legislation and enforcement with regard to label content, it should be encouraged that dietary supplement products highlight the message that there is the potential for adverse events to occur. ${ }^{5}$ Warning statements pertaining to 'exclusion of use' and 'not a cure for disease states', and alerting consumers to the potential for the presence of banned substances, ${ }^{5}$ should be more pronounced. The role, therefore, of communication with the patient through the credibility of the physician would be enhanced in getting these messages across to the patient.

\section{Conclusion}

This paper has shown the imperative responsibility for healthcare providers to consider conscientiously, through appropriate and rigorous assessment, whether a patient uses dietary supplements. Further, healthcare providers should have or develop an explicit understanding of the potential adverse effects of undeclared (on product labels) or embedded contaminants/adulterants in these products. This comprehension should align with the pledge in the historical Hippocratic Oath and this oath's modern-day successor. This pledge is aimed fundamentally at ensuring that the healthcare provider remains mindful of the need to maintain one's own health, well-being and abilities in order to provide care of the highest standard. In the interests of patient care, enquiry regarding the consumption of dietary supplement products is vitally important. Healthcare providers should be cognisant of the adverse effects profile, related patient history and possible drug-supplement interactions. Further, patients may embrace new lifestyle changes, which may alter their susceptibility to developing interactions. Such changes should be considered when a previously known patient presents to his/her provider with new, non-specific problems such as fatigue, fever and generalised pain. Sound healthcare provider-patient communication skills are therefore emphasised.

\section{Recommendation}

The authors would like to recommend that a formal legislative process is instituted for the healthcare fraternity to report and capture the incidence or observed incidences of adverse events potentially related to dietary supplements in a centralised database, which will improve vigilance and enforcement of such legislation. The ability of law enforcement to act timeously is required, such as the presence of early warning consumer communication systems and ensuring the potential recall of problem products in a speedy and efficient manner. Healthcare practitioners should receive information pertaining to dietary supplements problem products through regular policy briefs. Ongoing education and awareness campaigns aimed specifically at health practitioners should be provided. Formal training and/or attending accredited courses or workshops designed for graduate and postgraduate studies should be provided.

Acknowledgements - Gary Gabriels is a recipient of research grants from the South African Medical Research Council, the NRF, and forms part of a research team that receives NIHSS support.
Disclosure statement - No potential conflict of interest was reported by the authors.

\section{References}

1. Cook M. New Hippocratic Oath for doctors approved. 2017 [cited 2018 Nov 4]. https://www.bioedge.org/bioethics/new-hippocraticoath-for-doctors-approved/12496.

2. Hulkower R. The history of the hippocratic oath: outdated, inauthentic, and yet still relevant. Einstein J Biol Med. 2010: 41-44.

3. Parsa-Parsi RW. The revised declaration of Geneva - a modern-day physician's pledge. JAMA. 2017;318(20):1971-2.

4. Gabriels G, Mike Lambert M. Nutritional supplement products: does the label information influence purchasing decisions for the physically active. Nutr J. 2013;12:133.

5. Gabriels $G$, Lambert $M$, Smith P. Information on nutritional supplement labels: time for legislation? South Afr J Clin Nutr. 2012;25(1):22-6.

6. Chandra RV, Aruna CN, Nandakishore KJ, et al. Doctor-patient relationship - a review. IJOCR. 2013;1(1):11-3.

7. Lee $\mathrm{SJ}$, Back $A \mathrm{~L}$, Block SD, et al. Enhancing physician-patient communication. Am Soc Haematol - Haematol. 2002: 464-483.

8. Gabriels $G$, Lambert $M$, Smith $P$, et al. Fluoxetine contamination in dietary/nutritional supplements (un)bridges the quality of life for the youth to the elderly consumer. Med Res Arch. 2018;6(2):1-16.

9. Hjortdahl P. The influence of general practitioners' knowledge about their patients on the clinical decision-making process. Scand J Prim Health Care. 1992;10:290-4.

10. Verhaak PFM, Schellevis FG, Nuijen J, et al. Patients with a psychiatric disorder in general practice: determinants of general practitioners' psychological diagnosis. Gen Hosp Psychiatry. 2006;28:125-32.

11. Chatziefstathiou D, Talentino AK. Sporting boundaries, sporting events and commodification. Inter-Disciplinary Press; 2015. ISBN 978-1-84888-388-8

12. Dailey R, Schwartz KL, Binienda J, et al. Challenges in making therapeutic lifestyle changes among hypercholesterolemic AfricanAmerican patients and their physicians. J Natl Med Assoc. 2006;98 (12):1895-903.

13. Gabriels G, Smith P, Coopoo Y. South Africa perspectives for BRICS 'finger print' profiling of the trace and heavy metals in ginseng and hypoxis commercial available herbal products - a quality control tool. J Complement Med Alt Healthcare. 2017;4(5):555647. doi:10. 19080/ JCMAH.2017.04.55564.

14. Gabriels G, Lambert M, Smith $P$, et al. Fluoxetine contamination in dietary/nutritional supplements (un)bridges the quality of life for the youth to the elderly consumer. Med Res Arch. 2018;6:1-16.

15. Gabriels $G$, Lambert $M$, Smith $P$, et al. Melamine contamination in nutritional supplements - is it an alarm bell for the general consumer, athletes, and 'weekend warriors'? Nutr J. 2015;14:1-7.

16. O'Riordan $M$, et al. Dealing with uncertainty in general practice: an essential skill for the general practitioner. Qual Prim Care. 2011;19:175-81.

17. Wilkinson D, Stave C, Keohane D, et al. The role of general practitioners in the diagnosis and treatment of Alzheimer's disease: a multinational survey. J Int Med Res. 2004;32:149-59.

18. Block G, Jensen CD, Norkus EP, et al. Usage patterns, health, and nutritional status of long-term multiple dietary supplement users: a cross-sectional study. Nutr J. 2007;6:30. doi:10.1186/1475-2891-6-30.

19. Noorbhai $H$, Gabriels G, Noorbhai A. The effects of common medications in response to exercise and training. Adv Pharmacoepidemiol Drug Saf. 2014;3(1):1000146. ISSN: 2167-1052 APDS

20. Noorbhai $\mathrm{MH}$, Gabriels $\mathrm{G}$. The enterprising business of nutritional supplements: an eye-opener. Int J Drug Dev Res. 2016;8:042-3.

21. Creanor XM, Coopoo Y, Gabriels G. Attitudes towards nutritional supplement use amongst adult gymnasium users in Johannesburg North. South Afr J Sports Med. 2017;29:1-5. doi:10.17159/2078516X/2017/v29i0a1387.

22. Starzak DE, Derman W, McKune AJ, et al. Anti-doping knowledge and opinions of South African pharmacists and general practitioners. J Sports Med Doping Stud. 20166:181. doi:2161-0673.1000181. 